\title{
The Pricing of Dual-Expiry Exotics with Mean Reversion and Jumps
}

\author{
Kevin Z. Tong1,2, Dongping Hou², Jianhua Guan³ \\ ${ }^{1}$ Department of Mathematics and Statistics, University of Ottawa, Ottawa, Canada \\ ${ }^{2}$ Enterprise Risk and Portfolio Management, Bank of Montreal, Toronto, Canada \\ ${ }^{3}$ Equity Derivative Structuring, CIBC World Market Inc., Toronto, Canada \\ Email: ztong043@uottawa.ca, Dongping.hou@gmail.com, Jimjimjim0708@hotmail.com
}

How to cite this paper: Tong, K.Z., Hou, D.P. and Guan, J.H. (2019) The Pricing of Dual-Expiry Exotics with Mean Reversion and Jumps. Journal of Mathematical Finance, 9, 25-41.

https://doi.org/10.4236/jmf.2019.91003

Received: December 25, 2018

Accepted: January 26, 2019

Published: January 29, 2019

Copyright $\odot 2019$ by author(s) and Scientific Research Publishing Inc. This work is licensed under the Creative Commons Attribution International License (CC BY 4.0).

http://creativecommons.org/licenses/by/4.0/

\section{(c) (i) Open Access}

\begin{abstract}
This paper develops a new class of models for pricing dual-expiry options that are characterized by two expiry dates. The underlying asset price is modeled by a time changed exponential Ornstein Uhlenbeck (OU) process, where the time change process is a Lévy subordinator. The new models can capture both mean reversion and jumps often observed in various types of underlying assets of exotics. The pricing method exploits the observation that dual expiry options have payoffs that can be perfectly replicated by a particular set of first and second order binary options. The novelty of the paper is that we are able to derive the analytical solutions to the prices of these binaries through eigenfunction expansion method. Based on that, we can obtain the formulas for dual-expiry exotics through static replication. We also numerically investigate the sensitivities of prices of chooser, compound and extendable options with respect to the parameters of the models.
\end{abstract}

\section{Keywords}

Dual-Expiry Options, Binary Options, Eigenfunction Expansion, Lévy Subordinator, Stochastic Time Change, OU Process, Jump Diffusion

\section{Introduction}

Dual-expiry option is a class of exotic options that depends on a single underlying asset but whose payoff structure involves two fixed future dates. Usually, at the first expiry date, the option holder receives a contract that matures at the second expiry date. Chooser options, compound options, extendable options, shout options, American call options on an asset with a single known dividend payment and partial barrier options are good examples. The dual-expiry exotics 
can be easily generalized to multiple-expiry options, whose payoff structure involves several fixed future dates. Examples include ladder options, multiple shout options, Bermudan options, multiple extendable options and barrier options with discrete monitoring.

[1] develops a new technique for pricing dual-expiry exotic options by assuming the underlying asset price dynamics follows continuous geometric Brownian motion. The method exploits the observation that dual expiry options have payoffs that can be perfectly replicated by a particular set of first and second order binary options. Hence, in order to avoid arbitrage, the exotic option prices are obtained by static replication with respect to this family of binaries. As pointed out by [1], the representation of prices in terms of binaries is quite general and does not depend on any particular underlying asset price dynamics. In the Black-Scholes (BS) framework, [2] extends the method of [1] for pricing multiple-expiry options using a concept of higher order binary options. [3] [4] generalize [1] for dual- and multiple-expiry exotics to a Lévy environment. The binary option prices are derived using the mathematical methodology of [5] that employs the pseudo-differential operators whose symbol is expressed in terms of the characteristic exponent of the underly Lévy process. [6] further generalizes method of [1] to exotics with multiple-asset and multiple-expiry.

In this paper, we present a new class of models for pricing dual-expiry exotic options. We assume the underlying asset price is an exponential function of $\mathrm{OU}$ process subject to stochastic time change. The time change process is modeled by a Lévy subordinator. The resulting asset price then exhibits mean reversion and jumps.

We generalize the works of [1] [2] [3] [4] in two directions. In the previous works, the mean reversion is absent in the underlying asset price dynamics. As a result, these models are restricted to the dual-expiry exotics written on equities. On the other hand, the empirical evidence on mean reversion in financial asset is abundant. There is a census that many underlying assets of exotic option contracts, such as currencies, commodities, energy, temperature and even some stocks, display mean reversion. Our models are built on the OU process and can therefore be applied to a wider range of asset classes.

Time-changing a continuous time Markov process such as OU process can lead to a much wider class of models than classical jump-diffusion models. In the typical jump-diffusion models, such as [3] [4], the state-independent jumps are added to the diffusion process. In these models, upon arrival, the direction of the jump and the probability distribution of jump amplitude are independent of the current state of the process. Through Lévy subordination of OU process, our model, in contrast, can feature state-dependent mean reverting jumps with the jump direction and the jump amplitude dependent on the current state of the process. The subordinate OU process is found to be a better candidate for modeling phenomena where jumps depend on the state, such as mean-reverting jumps. See its successful applications for callable and putable bonds in [7], 
commodities in [8], electricity in [9] and variance swaps in [10]. We also refer to [11] for a brief introduction of subordinate Markov processes and [12] [13] [14] [15] for their further applications.

To solve the dual-expiry exotics pricing problems, we extend the works of [1] to the case where the asset price follows the mean reverting process with jumps. We are able to derive the analytical pricing formulas for first and second order binary options using eigenfunction expansion method. After that, we apply them to pricing of some dual-expiry exotics such as chooser options, compound options and extendable options by static replication. We need to emphasize that eigenfunction expansion method is particularly suitable for pricing contingent claims written on the subordinate processes. The subordinate process is as analytically tractable as the original process without time change. The subordinate process shares the same eigenfunctions with the original one and the only modification is the replacement of eigenvalues of the original process with the Laplace transform of the eigenvalues. To the best of our knowledge, this is the first piece of work that applies this method for pricing dual-expiry options and it can be extended with little effort to the case of multiple-expiry exotics. We refer to [11] [16] for the surveys on the eigenfunction expansion method and [7] [8] [9] [10] [12] [13] [14] [15] for its various applications.

The rest of the paper is organized as follows. In Section 2, we introduce the general framework for modeling asset price as a time changed exponential OU process, where the time change process is modeled by the Lévy subordinators. In Section 3, we introduce the eigenfunction expansion method for our new model and also discuss how to calculate some important integrals that are essential for the determination of the eigenfunction expansion coefficients. In Section 4, we apply the eigenfunction expansion method to the valuation of first and second order binary options. We provide the analytical formulas for these binaries. In Section 5, we express the payoffs of some dual-expiry options in terms of binary options and then demonstrate how to valuate these options by static replication. We also implement the model and analyze the effect of parameters of the model on the selected option prices through specific numerical examples.

\section{The Model Framework}

Let $(\Omega, \mathcal{F}, Q)$ be a probability space with an information filtration $\left(\mathcal{F}_{t}\right)$. Suppose under the risk neutral measure $Q$, the asset price process $S$ is governed by a time-changed exponential OU process, that is,

$$
S(t)=\exp (Y(t)),
$$

where

$$
Y(t)=X(T(t)),
$$

where $T$ is a time change process and $X$ is an OU process

$$
\mathrm{d} X(t)=\kappa(\theta-X(t)) \mathrm{d} t+\sigma \mathrm{d} B(t),
$$


where $\kappa>0$ and $B(t)$ is a standard Brownian motion.

To introduce jumps into the asset price dynamics, we will follow [7]-[15] and model the time change process $T$ as a Lévy subordinator. The Lévy subordinator $T$ is a nondecreasing process with positive jumps and non-negative drift with the Laplace transform:

$$
\mathrm{E}[\exp (-\lambda T(t))]=\exp (-t \phi(\lambda))
$$

where $\phi$ is the Lévy exponent and given by the Lévy-Khintchine formula (see e.g., [17])

$$
\phi(\lambda)=\gamma \lambda+\int_{(0, \infty)}(1-\exp (-\lambda s)) v(\mathrm{~d} s),
$$

where $\gamma \geq 0$ and the Lévy measure $v$ must satisfy

$$
\int_{(0, \infty)}(s \wedge 1) v(\mathrm{~d} s)<\infty .
$$

To make sure that the expectation in (4) is finite for $\lambda \in \mathbb{R}$, we also impose the following restriction

$$
\int_{(0, \infty)} \exp (-\lambda s) v(\mathrm{~d} s)<\infty .
$$

An important sub-class of Lévy subordinators are the tempered stable subordinators. For such subordinators, the Lévy measure $v(s)$ is given by

$$
v(s)=C s^{-1-p} \exp (-\eta s),
$$

where $C>0$ and $\eta>0$. Important special cases are the Gamma subordinator with $p=0$, the IG subordinator with $p=\frac{1}{2}$ and the compound Poisson subordinator with $p=-1$ and $\eta>0$. For such subordinators, the Lévy exponent is given by

$$
\phi(\lambda)= \begin{cases}\gamma \lambda-C \Gamma(-p)\left[(\lambda+\eta)^{p}-\eta^{p}\right], & p \neq 0 \\ \gamma \lambda+C \log \left(1+\frac{\lambda}{\eta}\right), & p=0\end{cases}
$$

We can also reparameterize the exponent by setting

$$
\begin{cases}\vartheta=-C \Gamma(-p) p \eta^{p-1}, \omega=-C \Gamma(-p) p(1-p) \eta^{p-2}, & p \neq 0 \\ \vartheta=\frac{C}{\eta}, \omega=\frac{C}{\eta^{2}}, & p=0\end{cases}
$$

where $\vartheta=\mathrm{E}(T(1))-\gamma$ and $\omega=\operatorname{Var}(T(1))$.

According to [11], when the process $X$ is time changed by a Lévy subordinator $T$, the resulting process $Y$ will be a jump-diffusion process with mean-reverting diffusion drift and mean-reverting jumps if $\gamma>0$ or a pure jump process with mean-reverting jumps if $\gamma=0$.

\section{Eigenfunction Expansion Method}

For the OU process $X$ in (3), its infinitesimal generator $\mathcal{L}$ is defined by 


$$
\mathcal{L} f(x)=\kappa(\theta-x) f^{\prime}(x)+\frac{1}{2} \sigma^{2} f^{\prime \prime}(x),
$$

where $f$ is transformation function. $f^{\prime}$ and $f^{\prime \prime}$ are first- and second-order derivatives of $f$, respectively.

Let $L^{2}((-\infty, \infty), m)$ denote the space of functions on $(-\infty, \infty)$ square integrable with the speed measure $m(\mathrm{~d} x)=m(x) \mathrm{d} x$ and endowed with the inner product $(f, g)$, where

$$
m(x)=\frac{2}{\sigma^{2}} \exp \left(-\frac{\kappa(\theta-x)^{2}}{\sigma^{2}}\right),
$$

and

$$
(f, g)=\int_{0}^{\infty} f(x) g(x) m(x) \mathrm{d} x .
$$

Then, for any $f \in L^{2}((-\infty, \infty), m)$, we have (see e.g., [11])

$$
\mathrm{E}[f(X(t)) \mid X(0)=x]=\sum_{n=0}^{\infty} f_{n} \exp \left(-\lambda_{n} t\right) \psi_{n}(x),
$$

where $f_{n}=\left(f, \psi_{n}\right),\left\{\lambda_{n}\right\}$ are the eigenvalues of $-\mathcal{L}$ and $\left\{\psi_{n}\right\}$ are the corresponding eigenfunctions satisfying the following Sturm-Liouville equation

$$
-\mathcal{L} \psi_{n}=\lambda_{n} \psi_{n} \text {. }
$$

For the OU process $X$ defined in (3), its eigenvalues and eigenfunctions can be summarized in the following result (see e.g., [11]):

Proposition 1 For the OU process $X$ defined in (3), the eigenvalues $\lambda_{n}$ and eigenfunction $\psi_{n}, n=0,1, \cdots$, are

$$
\lambda_{n}=\kappa n
$$

and

where $\xi=\frac{\sqrt{\kappa}}{\sigma}(x-\theta)$,

$$
\psi_{n}(x)=N_{n} H_{n}(\xi)
$$

$$
N_{n}^{2}=\frac{\sigma \sqrt{\kappa}}{2^{n+1} n ! \sqrt{\pi}}
$$

and $H_{n}(x)$ is the Hermite polynomial defined as

$$
H_{n}(x)=n ! \sum_{m=0}^{\left\lfloor\frac{n}{2}\right\rfloor} \frac{(-1)^{m}}{m !(n-2 m) !}(2 x)^{n-2 m} \text {. }
$$

For the time changed OU process $Y$ defined in (2), for any $f \in L^{2}((0, \infty), m)$, we can also employ the eigenfunction expansion method to compute the following expectation (see e.g., [8]):

$$
\begin{aligned}
\mathrm{E}[f(Y(t)) \mid X(0)=x] & =\sum_{n=0}^{\infty} f_{n} \mathrm{E}\left[\exp \left(-\lambda_{n} T(t)\right)\right] \psi_{n}(x) \\
& =\sum_{n=0}^{\infty} f_{n} \exp \left(-\phi\left(\lambda_{n}\right) t\right) \psi_{n}(x),
\end{aligned}
$$

where $\left\{\lambda_{n}\right\}$ and $\left\{\psi_{n}\right\}$ are the eigenvalues and eigenfunctions of OU process 
and can be obtained from (10) and (11), respectively. $\phi$ is the Lévy exponent for the time change process $T$.

It is clear that the eigenfunction expansion of $Y$ has the same form as $X$, but with $\exp \left(-\lambda_{n} t\right)$ replaced by $\exp \left(-\phi\left(\lambda_{n}\right) t\right)$. Thus, the eigenfunction expansion method makes the time changed model as tractable as the original model. This important result explains why eigenfunction expansion method is the natural way of computing option prices for the time-changed processes.

For our new pricing model, the eigenvalues and eigenfunctions of OU process can be calculated easily from Proposition 1. To employ the eigenfunction expansion method to calculate the dual-expiry exotics prices, we still need to obtain the eigenfunction expansion coefficient $f_{n}$. In this section, we provide the formulas for several integrals that will later be employed to calculate $f_{n}$.

The following formulas can be found in [18]:

\section{Lemma 1}

1) For $y>0$,

$$
\bar{\alpha}_{n}(y):=\int_{-\infty}^{\infty} \exp \left(-(x-y)^{2}\right) H_{n}(x) \mathrm{d} x=\sqrt{\pi}(2 y)^{n} .
$$

2)

$$
\bar{\beta}_{n}:=\int_{-\infty}^{\infty} \exp \left(-x^{2}\right) H_{n}(x) \mathrm{d} x= \begin{cases}\sqrt{\pi}, & n=0 \\ 0, & n \neq 0\end{cases}
$$

3)

$$
\bar{\gamma}_{m, n}:=\int_{-\infty}^{\infty} \exp \left(-x^{2}\right) H_{m}(x) H_{n}(x) \mathrm{d} x= \begin{cases}\sqrt{\pi} 2^{n} n !, & m=n \\ 0, & m \neq n\end{cases}
$$

The following integrals can be computed using the results of [7]:

\section{Lemma 2}

1) Define

$$
\alpha_{n}(c, u):=\int_{-\infty}^{u} \exp \left(c x-x^{2}\right) H_{n}(x) \mathrm{d} x .
$$

Then, $\alpha_{n}(c, u)$ can be computed recursively as follows:

$$
\alpha_{0}(c, u)=\frac{1}{2} \exp \left(\frac{c}{4}\right) \sqrt{\pi}\left(\operatorname{erf}\left(\frac{1}{2}(2 u-c)\right)+1\right),
$$

where $\operatorname{erf}(x)$ is the error function defined by

$$
\operatorname{erf}(x)=\frac{1}{\sqrt{\pi}} \int_{-\infty}^{x} \exp \left(-t^{2}\right) \mathrm{d} t,
$$

and for $n>0$,

$$
\alpha_{n}(c, u)=-\exp \left(c u-u^{2}\right) H_{n-1}(u)+c \alpha_{n-1}(c, u) .
$$

2)

$$
\beta_{n}(u):=\int_{-\infty}^{u} \exp \left(-x^{2}\right) H_{n}(x) \mathrm{d} x= \begin{cases}\sqrt{\pi} \Phi(\sqrt{2} u), & n=0 \\ -H_{n-1}(u) \exp \left(-u^{2}\right), & n \neq 0\end{cases}
$$


where $\Phi(x)$ is the CDF of standard normal distribution.

3) Define

$$
\gamma_{m, n}(u):=\int_{-\infty}^{u} \exp \left(-x^{2}\right) H_{m}(x) H_{n}(x) \mathrm{d} x .
$$

Then, $\gamma_{m, n}(u)$ can be calculated recursively as follows:

$$
\gamma_{0,0}(u)=\sqrt{\pi} \Phi(\sqrt{2} u), \gamma_{n, n}(u)=2 n \gamma_{n-1, n-1}(u)-H_{n-1}(u) H_{n}(u) \exp \left(-u^{2}\right), n \geq 1
$$

and for $m \neq n$,

$$
\gamma_{m, n}(u)=\frac{H_{m}(u) H_{n+1}(u)-H_{n}(u) H_{m+1}(u)}{2(n-m)} \exp \left(-u^{2}\right)
$$

\section{Valuation of First and Second Order Binaries}

Following [1], we define the first order up (or down) binary option as the option that delivers an agreed payoff on expiry date if the price of underlying asset is above (or below) a fixed exercise price and zero otherwise. Let $Y$ be the time changed OU process defined in (2). Let $s=(+,-)$ be sign indicators for the up and down binaries, respectively. Let $V_{\xi}^{s}\left(y, t, t_{0}\right)$ denote the time $t$ value of binaries with payoff function $f\left(Y\left(t_{0}\right)\right) 1_{\left\{s Y\left(t_{0}\right)>s \xi\right\}}$ at time $t_{0}, t_{0}>t$, conditioning on $Y(t)=y$, that is,

$$
V_{\xi}^{s}\left(y, t, t_{0}\right)=\exp \left(-r\left(t_{0}-t\right)\right) \mathrm{E}\left[f\left(Y\left(t_{0}\right)\right) 1_{\left\{s Y\left(t_{0}\right)>s \xi\right\}} \mid \mathcal{F}_{t}\right] .
$$

Clearly, the up and down binaries satisfy the parity relation

$$
V_{\xi}^{+}\left(y, t, t_{0}\right)+V_{\xi}^{-}\left(y, t, t_{0}\right)=\exp \left(-r\left(t_{0}-t\right)\right) \mathrm{E}\left[f\left(Y\left(t_{0}\right)\right) \mid \mathcal{F}_{t}\right] .
$$

We also denote three specific options as follows.

- $A_{\xi}^{s}\left(y, t, t_{0}\right)$ is the asset binary with $f(x)=\exp (x)$.

- $B_{\xi}^{s}\left(y, t, t_{0}\right)$ is the bond binary with $f(x)=1$.

- $Q_{\xi}^{s}\left(y, t, t_{0} ; k\right)$ is the Q-option with $f(x)=s(\exp (x)-k)$.

In the following theorem, we can derive the analytical formulas for the above three option prices.

Proposition 2 Assume the processes for the underlying asset price are given by (1)-(3). Let T be a Lévy subordinator with Lévy exponent $\phi$. Then,

1)

$$
A_{\xi}^{s}\left(y, t, t_{0}\right)=\exp \left(-r\left(t_{0}-t\right)\right) \sum_{n=0}^{\infty} f_{n}^{s}(\xi) \exp \left(-\phi\left(\lambda_{n}\right)\left(t_{0}-t\right)\right) \psi_{n}(y),
$$

where $\lambda_{n}$ and $\psi_{n}(y)$ are in (10) and (11), respectively. Furthermore,

$$
f_{n}^{-}(\xi)=\frac{2 N_{n}}{\sigma \sqrt{\kappa}} \exp (\theta) \alpha_{n}\left(\frac{\sigma}{\sqrt{\kappa}}, \frac{\sqrt{\kappa}}{\sigma}(\xi-\theta)\right),
$$

and

$$
f_{n}^{+}(\xi)=\frac{2 N_{n}}{\sigma \sqrt{\kappa}} \exp \left(\theta+\frac{\sigma^{2}}{4 \kappa}\right) \bar{\alpha}_{n}\left(\frac{\sigma}{2 \sqrt{\kappa}}\right)-f_{n}^{-}(\xi),
$$


where $\bar{\alpha}_{n}$ and $\alpha_{n}$ can be calculated using Lemma 1 and Lemma 2, respectively.

2)

$$
B_{\xi}^{s}\left(y, t, t_{0}\right)=\exp \left(-r\left(t_{0}-t\right)\right) \sum_{n=0}^{\infty} g_{n}^{s}(\xi) \exp \left(-\phi\left(\lambda_{n}\right)\left(t_{0}-t\right)\right) \psi_{n}(y)
$$

where

$$
g_{n}^{-}(\xi)=\frac{2 N_{n}}{\sigma \sqrt{\kappa}} \beta_{n}\left(\frac{\sqrt{\kappa}}{\sigma}(\xi-\theta)\right)
$$

and

$$
g_{n}^{+}(\xi)=\frac{2 N_{n}}{\sigma \sqrt{\kappa}} \bar{\beta}_{n}-g_{n}^{-}(\xi),
$$

where $\bar{\beta}_{n}$ and $\beta_{n}$ can be calculated using Lemma 1 and Lemma 2, respectively.

3)

$$
Q_{\xi}^{s}\left(y, t, t_{0} ; k\right)=s\left[A_{\xi}^{s}\left(y, t, t_{0}\right)-k B_{\xi}^{s}\left(y, t, t_{0}\right)\right] .
$$

Proof. To prove 1), using eigenfunction expansion, we have

$$
\begin{aligned}
A_{\xi}^{s}\left(y, t, t_{0}\right) & =\exp \left(-r\left(t_{0}-t\right)\right) \mathrm{E}\left[\exp \left(Y\left(t_{0}\right)\right) 1_{\left\{s Y\left(t_{0}\right)>s \xi\right\}} \mid \mathcal{F}_{t}\right] \\
& =\exp \left(-r\left(t_{0}-t\right)\right) \sum_{n=0}^{\infty} f_{n}^{s}(\xi) \exp \left(-\phi\left(\lambda_{n}\right)\left(t_{0}-t\right)\right) \psi_{n}(y),
\end{aligned}
$$

where

$$
\begin{aligned}
f_{n}^{-}(\xi) & =\int_{-\infty}^{\xi} \exp (x) \psi_{n}(x) m(x) \mathrm{d} x \\
& =\frac{2 N_{n}}{\sigma \sqrt{\kappa}} \int_{-\infty}^{\sqrt{\kappa}(\xi-\theta) / \sigma} \exp \left(\frac{\sigma}{\sqrt{\kappa}} y+\theta\right) H_{n}(y) \exp \left(-y^{2}\right) \mathrm{d} y \\
& =\frac{2 N_{n}}{\sigma \sqrt{\kappa}} \exp (\theta) \int_{-\infty}^{\sqrt{\kappa}(\xi-\theta) / \sigma} \exp \left(\frac{\sigma}{\sqrt{\kappa}} y-y^{2}\right) H_{n}(y) \mathrm{d} y \\
& =\frac{2 N_{n}}{\sigma \sqrt{\kappa}} \exp (\theta) \alpha_{n}\left(\frac{\sigma}{\sqrt{\kappa}}, \frac{\sqrt{\kappa}}{\sigma}(\xi-\theta)\right),
\end{aligned}
$$

where the equality in the last line comes from Lemma 2.

For $f_{n}^{+}(\xi)$, using Lemma 1, we obtain

$$
\begin{aligned}
f_{n}^{+}(\xi) & =\int_{\xi}^{\infty} \exp (x) \psi_{n}(x) m(x) \mathrm{d} x \\
& =\int_{-\infty}^{\infty} \exp (x) \psi_{n}(x) m(x) \mathrm{d} x-f_{n}^{-}(\xi) \\
& =\frac{2 N_{n}}{\sigma \sqrt{\kappa}} \exp (\theta) \int_{-\infty}^{\infty} \exp \left(-\left(y-\frac{\sigma}{2 \sqrt{\kappa}}\right)^{2}+\frac{\sigma^{2}}{4 \kappa}\right) H_{n}(y) \mathrm{d} y-f_{n}^{-} \\
& =\frac{2 N_{n}}{\sigma \sqrt{\kappa}} \exp \left(\theta+\frac{\sigma^{2}}{4 \kappa}\right) \bar{\alpha}_{n}\left(\frac{\sigma}{2 \sqrt{\kappa}}\right)-f_{n}^{-}(\xi) .
\end{aligned}
$$

The proof for 2) is similar to 1). The only differences are that we need to compute 


$$
g_{n}^{-}(\xi)=\int_{-\infty}^{\xi} \psi_{n}(x) m(x) \mathrm{d} x, g_{n}^{+}(\xi)=\int_{\xi}^{\infty} \psi_{n}(x) m(x) \mathrm{d} x
$$

which can both be analytically solved using Lemma 1 and Lemma 2.

3) can be obtained using the definition of Q-options.

We also define the second order binary option as an expiry $t_{0}$ binary contract on an underlying expiry $t_{1}$ binary option, $t_{0}<t_{1}$. Let $F_{\xi_{1}}^{s_{1}}\left(Y\left(t_{0}\right), t_{0}, t_{1}\right)$ denote a first order binary, $s_{0}, s_{1}$ be up-down indicators at times $t_{0}$ and $t_{1}$, respectively and $\xi_{0}, \xi_{1}$ be their corresponding exercise prices. The time $t$ value of the second order binaries, conditioning on $Y(t)=y$, is given by

$$
F_{\xi_{0} \xi_{1}}^{s_{0} s_{1}}\left(y, t, t_{0}, t_{1}\right)=\exp \left(-r\left(t_{0}-t\right)\right) \mathrm{E}\left[F_{\xi_{1}}^{s_{1}}\left(Y\left(t_{0}\right), t_{0}, t_{1}\right) 1_{\left\{s_{0} Y\left(t_{0}\right)>s_{0} \xi_{0}\right\}} \mid \mathcal{F}_{t}\right] .
$$

It is clear that the following up-down parity relation exists:

$$
F_{\xi_{0} \xi_{1}}^{+s_{1}}\left(y, t, t_{0}, t_{1}\right)+F_{\xi_{0} \xi_{1}}^{-s_{1}}\left(y, t, t_{0}, t_{1}\right)=F_{\xi_{1}}^{s_{1}}\left(y, t, t_{1}\right) .
$$

Depending on if the first order binary is an asset binary, a bond binary or a Q-option, we can define the corresponding second order binaries. We derive the analytical formulas for the second order binaries in the following theorem.

Proposition 3. Assume the processes for the underlying asset price are given by(1)-(3). Let T be a Lévy subordinator with Lévy exponent $\phi$. Then,

1)

$$
\begin{aligned}
A_{\xi_{0} \xi_{1}}^{s_{0} s_{1}}\left(y, t, t_{0}, t_{1}\right)= & \exp \left(-r\left(t_{1}-t\right)\right) \sum_{n=0}^{\infty} f_{n}^{s_{1}}\left(\xi_{1}\right) \exp \left(-\phi\left(\lambda_{n}\right)\left(t_{1}-t_{0}\right)\right) \\
& \times \sum_{m=0}^{\infty} h_{m n}^{s_{0}}\left(\xi_{0}\right) \exp \left(-\phi\left(\lambda_{m}\right)\left(t_{0}-t\right)\right) \psi_{m}(y),
\end{aligned}
$$

where functions $f_{n}^{s}$ is given in Proposition 2 and $\lambda_{n}$ and $\psi_{n}(y)$ are in (10) and (11), respectively. Furthermore,

$$
h_{m n}^{-}(\xi)=\frac{2 N_{m} N_{n}}{\sigma \sqrt{\kappa}} \gamma_{m, n}\left(\frac{\sqrt{\kappa}}{\sigma}(\xi-\theta)\right),
$$

and

$$
h_{m n}^{+}(\xi)=\frac{2 N_{m} N_{n}}{\sigma \sqrt{\kappa}} \bar{\gamma}_{m, n}-h_{m n}^{-}(\xi),
$$

where $\bar{\gamma}_{m, n}$ and $\gamma_{m, n}$ can be calculated using Lemma 1 and Lemma 2, respectively.

2)

$$
\begin{aligned}
B_{\xi_{0} \xi_{1}}^{s_{0} s_{1}}\left(y, t, t_{0}, t_{1}\right)= & \exp \left(-r\left(t_{1}-t\right)\right) \sum_{n=0}^{\infty} g_{n}^{s_{1}}\left(\xi_{1}\right) \exp \left(-\phi\left(\lambda_{n}\right)\left(t_{1}-t_{0}\right)\right) \\
& \times \sum_{m=0}^{\infty} h_{m n}^{s_{0}}\left(\xi_{0}\right) \exp \left(-\phi\left(\lambda_{m}\right)\left(t_{0}-t\right)\right) \psi_{m}(y)
\end{aligned}
$$

where $g_{n}^{s}$ is given in Proposition 2.

3)

$$
Q_{\xi_{0} \xi_{1}}^{s_{0} s_{1}}\left(y, t, t_{0}, t_{1} ; k\right)=s_{1}\left[A_{\xi_{0} \xi_{1}}^{s_{0} s_{1}}\left(y, t, t_{0}, t_{1}\right)-k B_{\xi_{0} \xi_{1}}^{s_{0} s_{1}}\left(y, t, t_{0}, t_{1}\right)\right] .
$$


Proof. It suffices to prove 1). Using eigenfunction expansion and iterated conditional expectation, we have

$$
\begin{aligned}
& A_{\xi_{0} \xi_{1}}^{s_{1} s_{1}}\left(y, t, t_{0}, t_{1}\right) \\
= & \exp \left(-r\left(t_{0}-t\right)\right) \mathrm{E}\left[A_{\xi_{1}}^{s_{1}}\left(Y\left(t_{0}\right), t_{0}, t_{1}\right) 1_{\left\{s_{0} Y\left(t_{0}\right)>s_{0} \xi_{0}\right\}} \mid \mathcal{F}_{t}\right] \\
= & \exp \left(-r\left(t_{1}-t\right)\right) \mathrm{E}\left[\exp \left(Y\left(t_{1}\right)\right) 1_{\left\{s_{1} Y\left(t_{1}\right)>s_{1} \xi_{1}\right\}} 1_{\left\{s_{0} Y\left(t_{0}\right)>s_{0} \xi_{0}\right\}} \mid \mathcal{F}_{t}\right] \\
= & \exp \left(-r\left(t_{1}-t\right)\right) \mathrm{E}\left\{1_{\left\{s_{0} Y\left(t_{0}\right)>s_{0} \xi_{0}\right\}} \mathrm{E}\left[\exp \left(Y\left(t_{1}\right)\right) 1_{\left\{s_{1} Y\left(t_{1}\right)>s_{1} \xi_{1}\right\}} \mid \mathcal{F}_{t_{0}}\right] \mid \mathcal{F}_{t}\right\} \\
= & \exp \left(-r\left(t_{1}-t\right)\right) \mathrm{E}\left\{1_{\left\{s_{0} Y\left(t_{0}\right)>s_{0} \xi_{0}\right\}} \sum_{n=0}^{\infty} f_{n}^{s_{1}}\left(\xi_{1}\right) \exp \left(-\phi\left(\lambda_{n}\right)\left(t_{1}-t_{0}\right)\right) \psi_{n}\left(Y\left(t_{0}\right)\right) \mid \mathcal{F}_{t}\right\} \\
= & \exp \left(-r\left(t_{1}-t\right)\right) \sum_{n=0}^{\infty} f_{n}^{s_{1}}\left(\xi_{1}\right) \exp \left(-\phi\left(\lambda_{n}\right)\left(t_{1}-t_{0}\right)\right) \\
& \times \sum_{m=0}^{\infty} h_{m n}^{s_{0}}\left(\xi_{0}\right) \exp \left(-\phi\left(\lambda_{m}\right)\left(t_{0}-t\right)\right) \psi_{m}(y),
\end{aligned}
$$

where

$$
\begin{aligned}
h_{m n}^{-}(\xi) & =\int_{-\infty}^{\xi} \psi_{m}(x) \psi_{n}(x) m(x) \mathrm{d} x \\
& =\frac{2 N_{m} N_{n}}{\sigma \sqrt{\kappa}} \int_{-\infty}^{\sqrt{\kappa}(\xi-\theta) / \sigma} \exp \left(-y^{2}\right) H_{m}(y) H_{n}(y) \mathrm{d} y \\
& =\frac{2 N_{m} N_{n}}{\sigma \sqrt{\kappa}} \gamma_{m, n}\left(\frac{\sqrt{\kappa}}{\sigma}(\xi-\theta)\right),
\end{aligned}
$$

and

$$
\begin{aligned}
h_{m n}^{+}(\xi) & =\int_{\xi}^{\infty} \psi_{m}(x) \psi_{n}(x) m(x) \mathrm{d} x \\
& =\int_{-\infty}^{\infty} \psi_{m}(x) \psi_{n}(x) m(x) \mathrm{d} x-h_{m n}^{-}(\xi) \\
& =\frac{2 N_{m} N_{n}}{\sigma \sqrt{\kappa}} \int_{-\infty}^{\infty} \exp \left(-y^{2}\right) H_{m}(y) H_{n}(y) \mathrm{d} y-f_{n}^{-}(\xi) \\
& =\frac{2 N_{m} N_{n}}{\sigma \sqrt{\kappa}} \bar{\gamma}_{m, n}-h_{m n}^{-}(\xi) .
\end{aligned}
$$

\section{Examples}

Once we obtain the analytical formulas for the first and second order binary options, we can apply the theoretical framework for pricing dual-expiry options developed by [1]. We can express the payoffs of dual-expiry exotics in terms of a portfolio of elementary binary options. According to the principle of static replication, if the payoff of a European style derivative can be expressed as a portfolio of elementary contacts, then the arbitrary free price of the derivative is the present value of this portfolio. Hereby, we utilize the results of the previous section to the pricing of some dual-expiry exotics.

\subsection{Chooser Options}

These exotics give the holder at time $t_{0}$, the choice of either a European call 
option of strike $k_{c}$ and expiry $t_{c}$, or a European put option of strike $k_{p}$ and expiry $t_{p}$. The payoff at time $t_{0}$, conditioning on $Y\left(t_{0}\right)=y\left(t_{0}\right)$, is therefore

$$
\begin{aligned}
V\left(t_{0}, y\left(t_{0}\right)\right) & =\max \left\{C\left(y\left(t_{0}\right), t_{0}, t_{c} ; k_{c}\right), P\left(y\left(t_{0}\right), t_{0}, t_{p} ; k_{p}\right)\right\} \\
& =\max \left\{Q_{\tilde{k}_{c}}^{+}\left(y\left(t_{0}\right), t_{0}, t_{c} ; k_{c}\right), Q_{\tilde{k}_{p}}^{-}\left(y\left(t_{0}\right), t_{0}, t_{p} ; k_{p}\right)\right\},
\end{aligned}
$$

where $C$ and $P$ are the call and put options, respectively. And $\tilde{k}_{c}=\log \left(k_{c}\right)$ and $\tilde{k}_{p}=\log \left(k_{p}\right)$.

We can calculate time $t$ value of chooser options from the following result.

Lemma 3 Assume the processes for the underlying asset price are given by (1)-(3). Let $T$ be a Lévy subordinator with Lévy exponent $\phi$. Then, the time $t$ value of chooser option, conditioning on $Y(t)=y$, is

$$
V(t, y)=Q_{d \tilde{k}_{c}}^{++}\left(y, t, t_{0}, t_{c} ; k_{c}\right)+Q_{d \tilde{k}_{p}}^{--}\left(y, t, t_{0}, t_{p} ; k_{p}\right),
$$

where $d$ is the unique solution of

$$
Q_{\tilde{k}_{c}}^{+}\left(d, t_{0}, t_{c} ; k_{c}\right)=Q_{\tilde{k}_{p}}^{-}\left(d, t_{0}, t_{p} ; k_{p}\right) .
$$

Proof. Since the call option function $Q_{\tilde{k}_{c}}^{+}\left(y\left(t_{0}\right), t_{0}, t_{c} ; k_{c}\right)$ is a monotonic increasing function of $y\left(t_{0}\right)$ and the put option function $Q_{\tilde{k}_{p}}^{-}\left(y\left(t_{0}\right), t_{0}, t_{p} ; k_{p}\right)$ is a monotonic decreasing function of $y\left(t_{0}\right)$, we will have a unique solution $d$ to (32). Then the value of chooser option at time $t$ is given by

$$
\begin{aligned}
V(t, y)= & \exp \left(-r\left(t_{0}-t\right)\right) \mathrm{E}\left[Q_{\tilde{k}_{c}}^{+}\left(y\left(t_{0}\right), t_{0}, t_{c} ; k_{c}\right) 1_{\left\{Y\left(t_{0}\right)>d\right\}}\right. \\
& \left.+Q_{\tilde{k}_{p}}^{-}\left(y\left(t_{0}\right), t_{0}, t_{p} ; k_{p}\right) 1_{\left\{Y\left(t_{0}\right)<d\right\}} \mid \mathcal{F}_{t}\right] \\
= & Q_{d \tilde{k}_{c}}^{++}\left(y, t, t_{0}, t_{c} ; k_{c}\right)+Q_{d \tilde{k}_{p}}^{--}\left(y, t, t_{0}, t_{p} ; k_{p}\right) .
\end{aligned}
$$

\subsection{Compound Options}

For these exotics, the underlying are options. There are four basic types of compound options, generally referred to as call-on-call, call-on-put, put-on-all and put-on-put options. At time $t_{0}$, the holder of a compound option has the right to buy (or sell) a standard European call (or put) option with strike price $k_{1}$ and expiry $t_{1}$ for price $k_{0}$. Let $s_{0}, s_{1}=(+,-)$ for call or put at time $t_{0}$ and $t_{1}$. Then the time $t$ value of a $s_{0} s_{1}$-compound option can be obtained from the following result.

Lemma 4 Assume the processes for the underlying asset price are given by (1)-(3). Let T be a Lévy subordinator with Lévy exponent $\phi$. Then, the time $t$ value of compound option, conditioning on $Y(t)=y$, is

$$
V(t, y)=s_{0} Q_{k_{2} k_{1}}^{s_{2} s_{1}}\left(y, t, t_{0}, t_{1} ; k_{1}\right)-s_{0} k_{0} B_{k_{2}}^{s_{2}}\left(y, t, t_{0}\right),
$$

where $\tilde{k}_{1}=\log \left(k_{1}\right), \quad s_{2}=s_{0} s_{1}$ and $k_{2}$ is the unique solution of

$$
Q_{\vec{k}_{1}}^{s_{1}}\left(k_{2}, t_{0}, t_{1} ; k_{1}\right)=k_{0} .
$$

Proof. Since the function $Q_{\tilde{k}_{1}}^{s_{1}}\left(y, t_{0}, t_{1} ; k_{1}\right)$ is a monotonic increasing 
(decreasing) function of $y$ when $s_{1}=+\left(s_{1}=-\right)$, the solution $k_{2}$ to (34) will be unique. The value of compound option at time $t$ is given by

$$
\begin{aligned}
V(t, y) & =\exp \left(-r\left(t_{0}-t\right)\right) \mathrm{E}\left\{\left[s_{0}\left(Q_{\vec{k}_{1}}^{s_{1}}\left(Y\left(t_{0}\right), t_{0}, t_{1} ; k_{1}\right)-k_{0}\right)\right]^{+} \mid \mathcal{F}_{t}\right\} \\
& =\exp \left(-r\left(t_{0}-t\right)\right) \mathrm{E}\left\{\left[s_{0}\left(Q_{\tilde{k}_{1}}^{s_{1}}\left(Y\left(t_{0}\right), t_{0}, t_{1} ; k_{1}\right)-k_{0}\right)\right] 1_{\left\{s_{2} Y\left(t_{0}\right)>s_{2} k_{2}\right\}} \mid \mathcal{F}_{t}\right\} \\
& =s_{0} Q_{k_{2} s_{1}}^{s_{2} s_{1}}\left(y, t, t_{0}, t_{1} ; k_{1}\right)-s_{0} k_{0} B_{k_{2}}^{s_{2}}\left(y, t, t_{0}\right) .
\end{aligned}
$$

\subsection{Extendable Options}

These exotics allow expiry date to be extended to a future date for a fee with a different strike price at the extended expiry. The holder of an expendable call option has the right at time $t_{0}$ to exercise a standard European call option with strike price $k_{0}$; or for premium $p$, to extend the expiry date to time $t_{1}>t_{0}$ and change the strike from $k_{0}$ to $k_{1}$. The time $t$ value of an extendable option can be obtained using the following result.

Lemma 5. Assume the processes for the underlying asset price are given by (1)-(3). Let $T$ be a Lévy subordinator with Lévy exponent $\phi$. Then the time $t$ value of extendable option, conditioning on $Y(t)=y$, is

$$
\begin{aligned}
V(t, y)= & Q_{I_{2}}^{+}\left(y, t, t_{0} ; k_{0}-p\right)-p B_{I_{1}}^{+}\left(y, t, t_{0}\right) \\
& +Q_{I_{1} \tilde{k}_{1}}^{++}\left(y, t, t_{0}, t_{1} ; k_{1}\right)-Q_{I_{2} \tilde{k}_{1}}^{++}\left(y, t, t_{0}, t_{1} ; k_{1}\right),
\end{aligned}
$$

where $\tilde{k}_{1}=\log \left(k_{1}\right)$ and $I_{1}$ and $I_{2}$ are the solutions of

$$
Q_{\tilde{k}_{1}}^{+}\left(I_{1}, t_{0}, t_{1} ; k_{1}\right)=p \text {, }
$$

and

$$
Q_{\tilde{k}_{1}}^{+}\left(I_{2}, t_{0}, t_{1} ; k_{1}\right)=I_{2}+p-k_{0} .
$$

Proof. Assume that the $I_{1}$ and $I_{2}$ are the unique solutions to (36) and (37), respectively. Then the value of extendable option at time $t$ is given by

$$
\begin{aligned}
V(t, y)= & \exp \left(-r\left(t_{0}-t\right)\right) \mathrm{E}\left[\left(Q_{\tilde{k}_{1}}^{+}\left(Y\left(t_{0}\right), t_{0}, t_{1} ; k_{1}\right)-p\right)\left(1_{\left\{Y\left(t_{0}\right)>I_{1}\right\}}-1_{\left\{Y\left(t_{0}\right)>I_{2}\right\}}\right)\right. \\
& \left.+\left(\exp \left(Y\left(t_{0}\right)\right)-k_{0}\right) 1_{\left\{Y\left(t_{0}\right)>I_{2}\right\}} \mid \mathcal{F}_{t}\right] \\
= & \exp \left(-r\left(t_{0}-t\right)\right) \mathrm{E}\left[\left(\exp \left(Y\left(t_{0}\right)\right)-\left(k_{0}-p\right)\right) 1_{\left\{Y\left(t_{0}\right)>I_{2}\right\}} \mid \mathcal{F}_{t}\right] \\
& -\exp \left(-r\left(t_{0}-t\right)\right) p \mathrm{E}\left[1_{\left\{Y\left(t_{0}\right)>I_{1}\right\}} \mid \mathcal{F}_{t}\right] \\
+ & \mathrm{E}\left[Q_{\tilde{k}_{1}}^{+}\left(Y\left(t_{0}\right), t_{0}, t_{1} ; k_{1}\right)\left(1_{\left\{Y\left(t_{0}\right)>I_{1}\right\}}-1_{\left\{Y\left(t_{0}\right)>I_{2}\right\}}\right) \mid \mathcal{F}_{t}\right] \\
= & Q_{I_{2}}^{+}\left(y, t, t_{0} ; k_{0}-p\right)-p B_{I_{1}}^{+}\left(y, t, t_{0}\right)+Q_{I_{1} \tilde{k}_{1}}^{++}\left(y, t, t_{0}, t_{1} ; k_{1}\right) \\
& -Q_{I_{2} \tilde{k}_{1}}^{++}\left(y, t, t_{0}, t_{1} ; k_{1}\right) .
\end{aligned}
$$

\subsection{Numerical Analysis}

In this section, we numerically study chooser, compound and extendable options 
based on a specific time-changed process. We assume the time change process $T$ is a Gamma subordinator with the Lévy exponent

$$
\phi(\lambda)=\frac{\vartheta^{2}}{\omega} \log \left(1+\frac{\lambda \omega}{\vartheta}\right) .
$$

To calculate the prices for dual exotics, we need to truncate the eigenfunction expansion after a finite number of terms. Following [14], we truncate the infinite series when a given error tolerance level is reached. In practice, we find the convergence of the expansion is rather fast.

In Figures 1-3, we perform some sensitivity tests to demonstrate how sensitive the selected dual-expiry exotics are to the changes in the key parameters in the model. We can summarize the findings as follows:

- The option prices decrease with the mean reverting parameter $\kappa$ for chooser and compound options, but increase for extendable options.
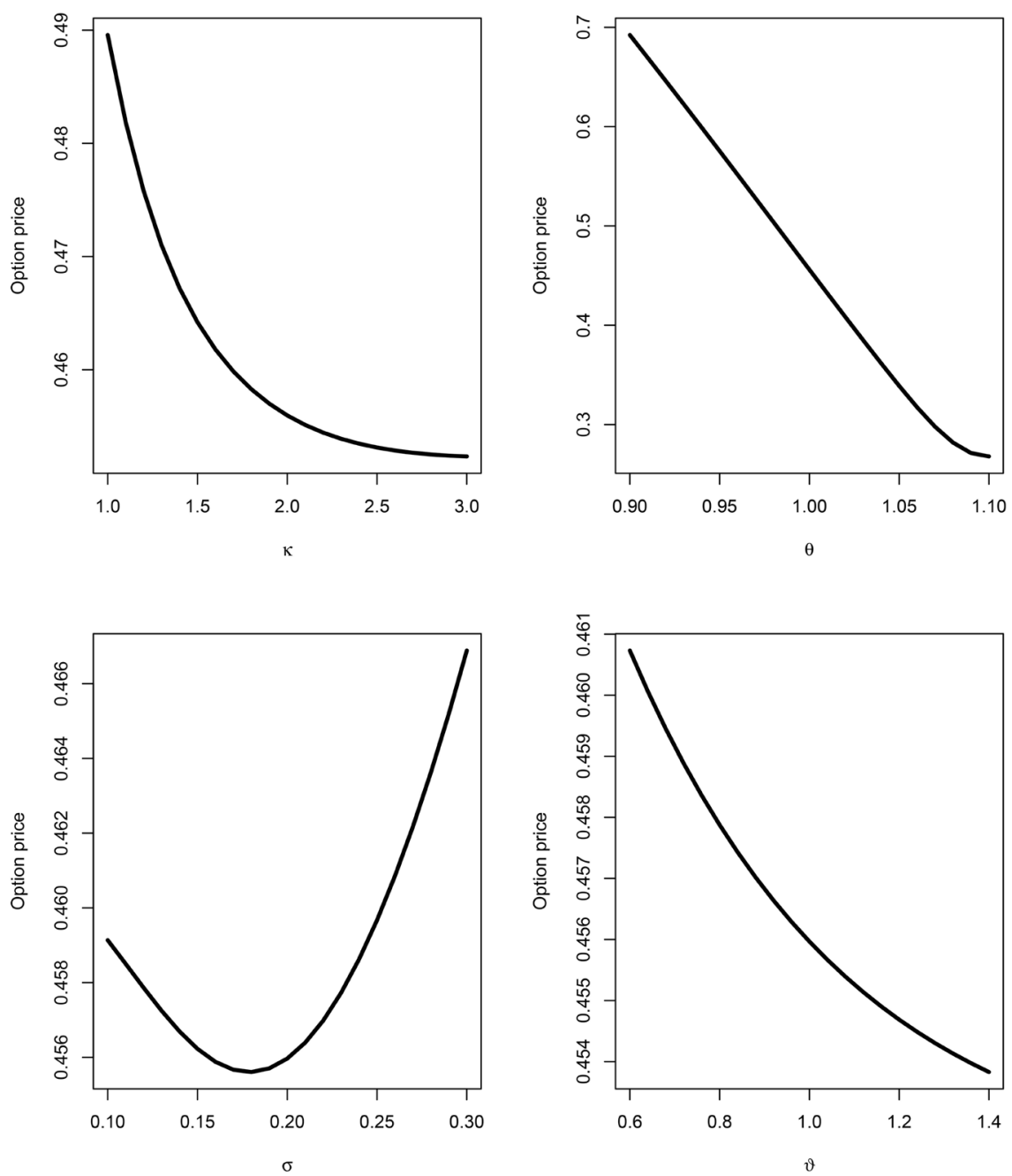

Figure 1. Sensitivities of chooser option prices to the parameters of the model. The parameters in the base case are $\kappa=2, \sigma=0.2, \theta=1, \vartheta=1, \omega=0.02, S_{0}=2.5$, $r=0.05, t=0, t_{0}=0.5, t_{c}=0.8, t_{p}=1, k_{c}=2.8$ and $k_{p}=3.2$. 
- The option prices are decreasing function of long-run mean parameter $\theta$ for chooser options but increasing for compound and extendable options.

- The option prices are monotonically increasing with asset variance parameter $\sigma$ for compound options, whereas the relationship between option prices and variance is $\mathrm{U}$-shaped for both chooser and extendable options.

- The option prices increase with the mean of Gamma subordinator $T(1)$ for both compound and extendable options but decrease for chooser options.

We note that the sensitivities of option prices to the model parameters depend on the option types. This is plausible for two reasons. First, different types of exotics respond differently with respect to the changes in the parameters. Second, the existence of mean reversion together with Lévy subordination can produce complex price dynamics. For example, when the mean reversion speed increases, the conditional mean of asset price will increase/decrease depending on if spot price is below/above the long-term mean, whereas there is no clear-cut relation
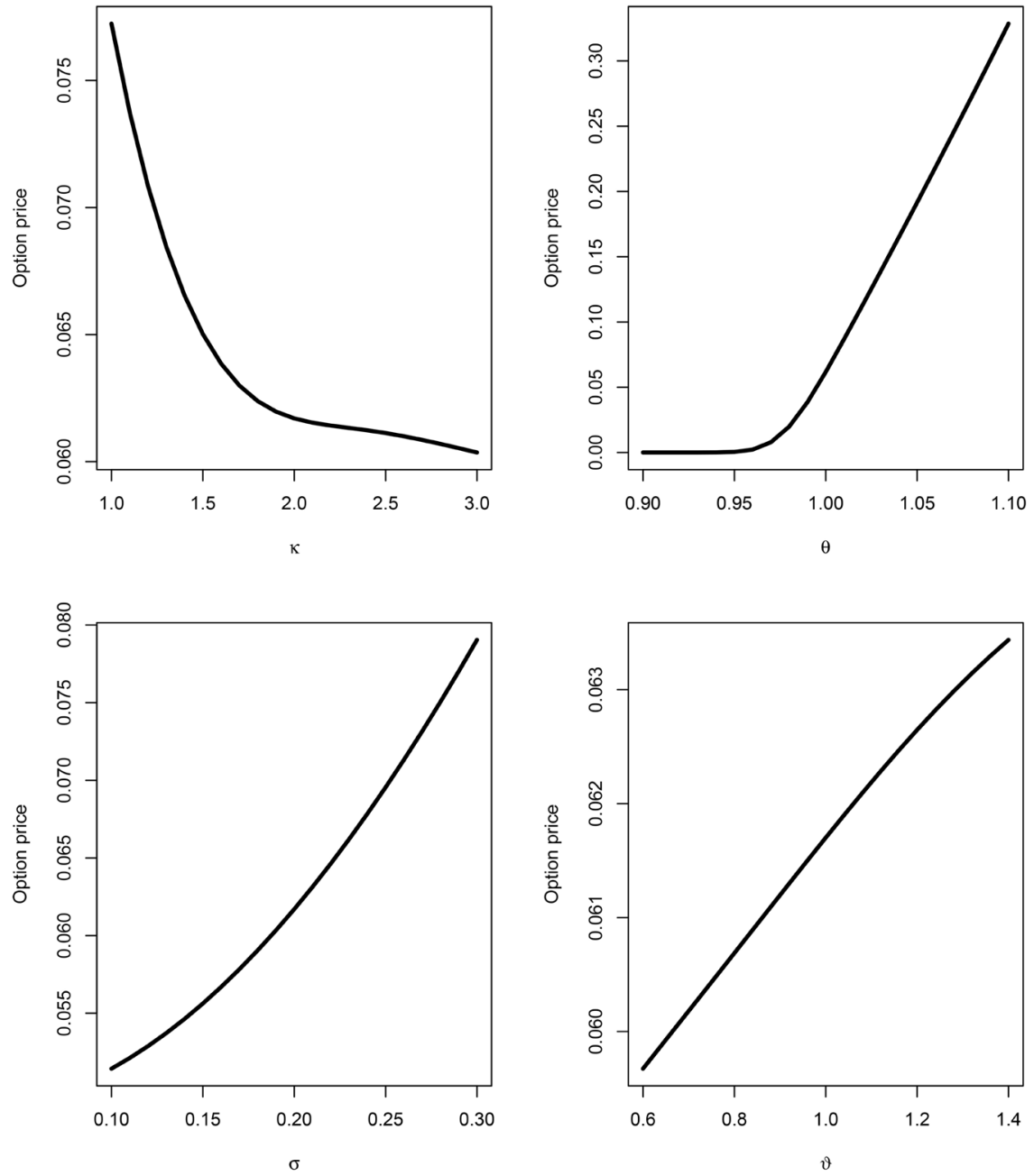

Figure 2. Sensitivities of compound option prices to the parameters of the model. The parameters in the base case are $\kappa=2, \sigma=0.2, \theta=1, \vartheta=1, \omega=0.02, S_{0}=2.5$, $r=0.05, t=0, t_{0}=0.5, t_{1}=1, k_{0}=2.5, k_{1}=0.1, s_{0}=1$ and $s_{1}=1$. 

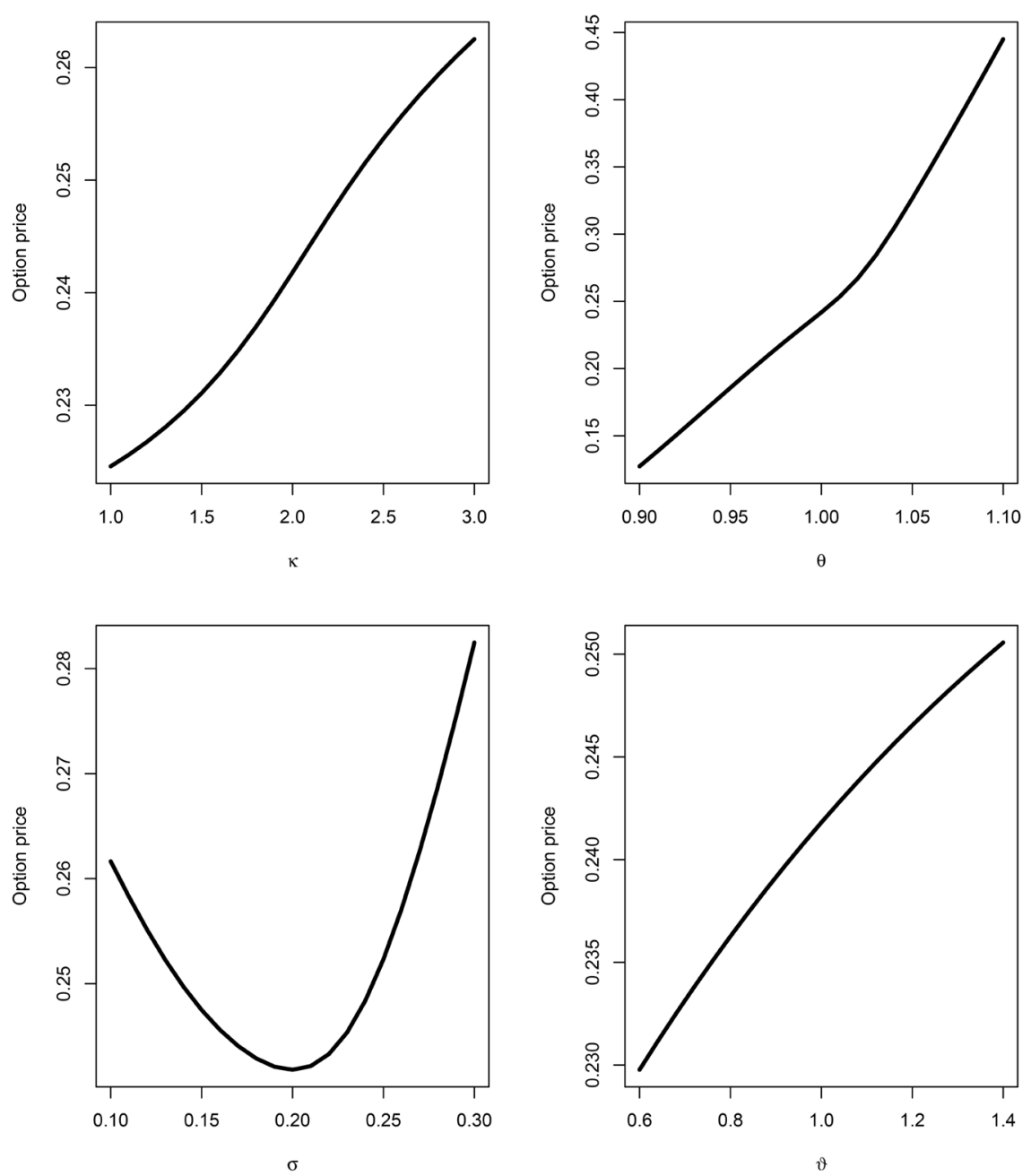

Figure 3. Sensitivities of extendable option prices to the parameters of the model. The parameters in the base case are $\kappa=2, \sigma=0.2, \theta=1, \vartheta=1, \omega=0.02, S_{0}=2.5$, $r=0.05, t=0, t_{0}=0.5, t_{1}=1, k_{0}=2.5, k_{1}=2.8$ and $p=0.1$.

between conditional variance and mean reversion speed. The subordination complicates the issue further. Therefore, it is not surprising to find the impacts of model parameters differ across the option types.

\section{Conclusion}

This paper studies a new class of models for pricing dual-expiry exotic options. The underlying asset price is modeled as exponential function of OU process time changed by a Lévy subordinator. The resulting asset price can display both mean reversion and jumps often observed in a large range of underlying assets of exotic option contracts. We employ the method of [1] to decompose the exotic option prices into a portfolio of first and second order binaries. We are able to employ the eigenfunction expansion technique to derive the analytical pricing formulas for the binaries. After that, we can compute dual-expiry exotics option prices by static replication. We also implement the model and analyze the effect 
of parameters of the model on some examples of exotics through specific numerical examples. We need to emphasize our techniques can be extended with minor changes when pricing multiple-expiry options.

\section{Conflicts of Interest}

The authors declare no conflicts of interest regarding the publication of this paper.

\section{References}

[1] Buchen, P.W. (2004) The Pricing of Dual-Expiry Exotics. Quantitative Finance, 4, 101-108. https://doi.org/10.1088/1469-7688/4/1/009

[2] O, H.C. and Kim, M.C. (2013) Higher Order Binary Options and Multiple-Expiry Exotics. Electronic Journal of Mathematical Analysis and Applications, 1, 247-259.

[3] Agliardi, R. (2009) The Quintessential Option Pricing Formula under Lévy Processes. Applied Mathematics Letters, 22, 1626-1631. https://doi.org/10.1016/j.aml.2009.05.008

[4] Agliardi, R. (2011) A Comprehensive Mathematical Approach to Exotic Option Pricing. Mathematical Methods in the Applied Sciences, 35, 1256-1268. https://doi.org/10.1002/mma.2519

[5] Boyarchenko, S.I. and Levendorskii, S.Z. (2002) Non-Gaussian Merton-Black-Scholes Theory. World Scientific, Singapore. https://doi.org/10.1142/4955

[6] Buchen, P.W. (2012) An Introduction to Exotic Option Pricing. CRC Press, Boca Raton. https://doi.org/10.1201/b11589

[7] Lim, D., Li, L. and Linetsky, V. (2012) Evaluating Callable and Putable Bonds: An Eigenfunction Expansion Approach. Journal of Economic Dynamics \& Control, 36, 1888-1908. https://doi.org/10.1016/j.jedc.2012.06.002

[8] Li, L. and Linetsky, V. (2014) Time-Changed Ornstein-Uhlenbeck Processes and Their Applications in Commodity Derivative Models. Mathematical Finance, 24, 289-330. https://doi.org/10.1111/mafi.12003

[9] Li, L., Mendoza-Arriaga, R., Mo, Z. and Mitchell, D. (2016) Modelling Electricity Prices: A Time Change Approach. Quantitative Finance, 16, 1089-1109. https://doi.org/10.1080/14697688.2015.1125521

[10] Tong, Z. and Liu, A. (2017) Analytical Pricing Formulas for Discretely Sampled Generalized Variance Swaps under Stochastic Time Change. International Journal of Financial Engineering, 4, 1-24. https://doi.org/10.1142/S2424786317500281

[11] Linetsky, V. and Mitchell, D. (2008) Spectral Methods in Derivatives Pricing, in Birge, J.R. and Linetsky, V. (editors). Handbook of Financial Engineering, Elsevier, Amsterdam, 223-299.

[12] Mendoza-Arriaga, R., Carr, P. and Linetsky, V. (2010) Time Changed Markov Processes in Unified Credit-Equity Modeling. Mathematical Finance, 20, 527-569. https://doi.org/10.1111/j.1467-9965.2010.00411.x

[13] Mendoza-Arriaga, R. and Linetsky, V. (2013) Time-Changed CIR Default Intensities with Two-Sided Mean-Reverting Jumps. The Annals of Applied Probability, 24, 811-856. https://doi.org/10.1214/13-AAP936

[14] Li, J., Li, L. and Zhang, G. (2017) Pure Jump Models for Pricing and Hedging VIX Derivatives. Journal of Economic Dynamics \& Control, 74, 28-55. 
https://doi.org/10.1016/j.jedc.2016.11.001

[15] Tong, Z. and Liu, A. (2018) Analytical Pricing of Discrete Arithmetic Asian Options under Generalized CIR with Time Change. International Journal of Financial Engineering, 5, 1-21. https://doi.org/10.1142/S2424786318500020

[16] Linetsky, V. (2004) The Spectral Decomposition of the Option Value. International Journal of Applied and Theoretical Finance, 5, 337-384. https://doi.org/10.1142/S0219024904002451

[17] Sato, K. (1999) Lévy Processes and Infinitely Divisible Distribution. Cambridge University Press, Cambridge.

[18] Prudnikov, A.P., Brychkov, Y.A. and Marichev, O.I. (1986) Integrals and Series, Vol. 2. Gordon and Breach Science Publishers, London. 